\title{
Tuning of salt separation efficiency by flow rate control in microfluidic dynamic dialysis
}

\author{
Prameen C. Kalikavunkal ${ }^{1} \cdot$ Nicolas G. Green $^{1}$ (D) - Maurits R. R. de Planque ${ }^{1}$ (D)
}

Received: 18 April 2019 / Accepted: 24 August 2019 / Published online: 11 September 2019

(c) The Author(s) 2019

\begin{abstract}
Microliter-scale separation processes are important for biomedical research and point-of-care diagnostics with small-volume clinical samples. Analytical assays such as mass spectrometry and field effect sensing necessitate sample desalting, but too low a salt concentration can disrupt protein structures and biomolecular interactions. In this work, we investigated whether salt extraction from a protein solution can be controlled by dynamic dialysis parameters. A microfluidic counter-flow dialyzer with a $5 \mathrm{kDa}$ molecular weight cut-off cellulose membrane was fabricated by laser cutting and operated with a wide range of feed and dialysis flow rates. It was found that with the appropriate flow conditions, most notably the feed flow rate, retentate salt concentrations from 0.1 to $99 \%$ of the input $\mathrm{NaCl}$ concentration can be achieved. The experimental data were in good agreement with a theoretical diffusion-based mass transfer model. The salt dialysis performance was similar in the presence of $50 \mathrm{mg} / \mathrm{mL}$ albumin, approximating blood plasma protein content, and did not deteriorate with overnight continuous dialysis, indicating minimal membrane fouling. The dialyzer construction method is compatible with all planar membranes, enabling implementation of tuneable dynamic dialysis for a wide range of on-line microfluidic biomolecular separations.
\end{abstract}

Keywords Dynamic dialysis $\cdot$ Desalting modulation $\cdot$ Microfluidics $\cdot$ Integrated membrane

\section{Introduction}

Membrane-facilitated molecular separations play a key role in many large-scale industrial processes, ranging from seawater desalination to fruit juice clarification, and also in milliliter-scale sample processing in biomedical research laboratories (van Reis and Zydney 2007; Saxena et al. 2009; Kazemi et al. 2016). Miniaturization towards microliter volumes can be achieved by integrating membranes with microfluidic systems (de Jong et al. 2006; Chen et al. 2016; Roelofs et al. 2015; Wardrip and Arnusch 2016; Han and Hwang 2018). Microfluidic chips also enable in situ microscopy of the membrane, e.g., to study membrane fouling, and electrical or optical characterization of the permeate and retentate phases, facilitating separation performance evaluation of novel membrane materials and transport mechanisms (Roelofs et al. 2015; Kwak et al. 2013; Kornreich et al. 2014). In ultrafiltration, e.g., for protein concentration

Maurits R. R. de Planque

m.deplanque@soton.ac.uk

1 Electronics and Computer Science, University of Southampton, Southampton SO17 1BJ, UK or buffer exchange, mass transport over the membrane is driven by pressure-induced flux; while mass transport in a dialysis process is primarily determined by concentration gradients over the membrane (Saxena et al. 2009).

Dialysis efficiency is significantly enhanced by minimizing the diffusion distance to the membrane and by maintaining a steep concentration gradient (Roelofs et al. 2015). Microdialysis flow cells offer the advantages of a sub-millimeter height of the feed channel and a continuous flow of fresh solution, buffer or pure water, through the dialysate channel. In a theoretical study, Yeh and Hsu evaluated the mass transfer efficiency for three different flow configurations (Yeh and Hsu 1999). Counter-current flow, where the sample and the dialysate phase flow in opposite directions along the membrane, gave the highest mass exchange; while co-current flow was the least effective. Cross-flow, implying that the dialysate stream is perpendicular to the sample stream (e.g., crossing a bundle of hollow fiber membranes), had an intermediate performance (Yeh and Hsu 1999). It was also demonstrated experimentally, for a flow cell with a planar microporous cellulose ester membrane, that countercurrent operation results in more efficient dialysis than cocurrent flow (Yeh and Chang 2005). 
Microfluidic dialysis cells have been described for a variety of applications, including small-molecule, peptide or protein extraction from protein mixtures (Perozziello et al. 2015), cell lysates (Xiang et al. 1999; Liu and Verma 1999) or blood (Kurita et al. 2006) for spectroscopic/spectrometric characterization, the exchange of metabolites and inhibitory species for cell-free protein synthesis (Timm et al. 2016), and the ionic strength modulation of oligonucleotide separation (Sheng and Bowser 2012) and virus phase transitions (Kornreich et al. 2014). Furthermore, many microfluidic devices, incorporating hollow fiber (Liu and Verma 1999; Canarelli et al. 2002; Jakubowski et al. 2005; Sun et al. 2008), planar polymer (Xu et al. 1998; Liu et al. 1998; Xiang et al. 1999) or planar inorganic (Tibavinsky et al. 2015; Wu et al. 2018) membranes, have been developed for the on-line desalting of biological samples for mass spectrometry analysis. The membrane pore size is always determined by the separation requirements, while the channel dimensions follow from the typical sample volume and the microfabrication methodology. The counter-flow configuration is most commonly employed, but the effect of the flow rate is typically not described, or is only investigated within a limited range, while the separation outcome tends to be assessed indirectly, for example as the signal-to-noise ratio in a mass spectrum (Xu et al. 1998; Liu and Verma 1999; Canarelli et al. 2002; Wu et al. 2018).

To establish to which extent the separation performance can be modulated by the hydrodynamic conditions, we quantified salt transfer over a regenerated cellulose membrane in a lasermachined microfluidic counter-flow dialyzer for a wide range of feed and dialysate flow rates. We obtained retentate salt concentrations between 0.1 and $99 \%$ of the input salt concentration, indicating that with the appropriate water and sample flow settings, any desired salt transfer efficiency can be realized. Theoretical mass transfer analysis for the counter-flow dialysis geometry closely matched the experimental observations, consistent with a diffusion-limited membrane transport mechanism. At a high protein concentration of $50 \mathrm{mg} / \mathrm{mL}$ albumin, dialysis performance was only marginally affected over a period of $16 \mathrm{~h}$, suggesting minimal fouling of the $5 \mathrm{kDa}$ MWCO cellulose membrane. Flow-controlled microfluidic diffusion dialysis, hence, enables applications such as tuneable desalting of biomolecular samples, where a physiological salt concentration interferes with analytical assays but too low a salt concentration induces protein denaturation or modifies biomolecular interactions (Xu et al. 1998; Zhou and Pang 2018).

\section{Experimental}

\subsection{Materials}

Planar regenerated cellulose and polyethersulfone ultrafiltration (UF) membranes with molecular weight cut-off (MWCO) values of 1, 5 and $10 \mathrm{kDa}$ were obtained from Sartorius Stedim Biotech as membrane disks of $25 \mathrm{~mm}$ diameter and $180 \mu \mathrm{m}$ thickness. Polycarbonate track-etch membrane filters of $25 \mathrm{~mm}$ diameter and $20 \mu \mathrm{m}$ thickness, with a $50 \mathrm{~nm}$ pore diameter and $1.18 \%$ porosity, were from Avanti Polar Lipids. Acrylic sheets of $5 \mathrm{~mm}$ thickness were purchased from RS Components. Sodium chloride and fluorescently (fluorescein isothiocyanate) labeled bovine serum albumin (BSA) were from Sigma Aldrich. Deionized water from a Milli-Q system was used for all experiments. Prior to use, to remove the humectant, the membranes were placed in a beaker containing deionized water for $1 \mathrm{~h}$, after which they were rinsed with fresh water and gently wiped with tissue paper.

\subsection{Membrane characterization}

Membrane thickness was verified by scanning electron microscopy of sputter-coated membrane cross sections with a Zeiss EVO LS25 microscope. The porosity of the UF membranes was determined by conductometry (Garg et al. 2012, 2014). After washing with deionized water, membranes were clamped between two Delrin chambers of $1 \mathrm{~mL}$ volume with a $5 \mathrm{~mm}$ diameter aperture in the side wall, where the membranes were in contact with an electrolyte solution of $10 \mathrm{mM} \mathrm{NaCl} . \mathrm{Ag} / \mathrm{AgCl}$ electrodes of $0.8 \mathrm{~mm}$ diameter were inserted in each chamber, DC voltages up to $1 \mathrm{~V}$ were applied and the current flowing over the membrane was measured. The conductance of the electrolyte-exposed membrane area was then determined from the $I-V$ relationship. The porosity of the UF membranes was estimated according to:

$P_{\mathrm{UF}}=P_{\text {ref }} \frac{L_{\mathrm{UF}} \times G_{\mathrm{UF}}}{L_{\mathrm{ref}} \times G_{\mathrm{ref}}}$,

where $G$ is the conductance, $L$ is the pore length (i.e., membrane thickness) and $P$ is the porosity of the membrane. From the conductance measurements of the UF and tracketch membranes, the porosity of the UF membranes can be approximated, neglecting the influence of pore geometry and assuming no distinct interactions of ions with the membrane material, using the known porosity and thickness of a tracketch membrane as reference values (Kumar and Chakarvarti 2008). 


\subsection{Counter-flow dialysis cell}

A microfluidic dialyzer was constructed by clamping a planar membrane between two optically transparent plates of poly(methyl methacrylate) (PMMA) with micromachined channels. Acrylic sheets of $5 \mathrm{~mm}$ thickness were cut into plates of $3 \times 3 \mathrm{~cm}$ with an Epilog Mini $30 \mathrm{~W} \mathrm{CO}_{2}$ laser cutter, and a screw hole was cut in each corner of the plate. In the top plate, a feed channel of $0.2 \mathrm{~mm}$ depth and $1.8 \mathrm{~mm}$ width was laser ablated, while a water channel of $3.5 \mathrm{~mm}$ depth and $3.0 \mathrm{~mm}$ width was defined in the bottom plate. Both channels are of spiral geometry, with a length of $\sim 75 \mathrm{~mm}$, giving a feed and a water channel volume of approximately 25 and $800 \mu \mathrm{L}$, respectively. These different dimensions imply a different relation between the volumetric flow rate and the linear velocity for the feed and water channels, as indicated in the text. At the start and end of each channel, a $0.8 \mathrm{~mm}$ diameter through-plate hole was laser machined as a tubing connector. A water-washed circular membrane sheet was positioned on the bottom plate and then the top plate was put into place. Both plates were brought into tight contact by fastening the M4 screws in the corners of the device. The membrane surface in contact with the feed channel is $\sim 1.35 \times 10^{-4} \mathrm{~m}^{2}$. Figure 2a shows an assembled dialyzer with connected microfluidic PTFE tubing (inner and outer diameter $0.3 \mathrm{~mm}$ and $0.8 \mathrm{~mm}$, respectively). Counterflow dialysis was implemented with Harvard Apparatus 11 Plus syringe pumps, with the feed and the water phase flowing in opposite directions along the membrane. The conductivity of aliquots of the retentate outflow was measured with a Horiba B-173 conductivity meter with a $100 \mu \mathrm{L}$ sample compartment and converted to $\mathrm{NaCl}$ concentration by a calibration curve. All experiments were performed at room temperature.

\subsection{Mass transfer analysis}

For urea dialysis over a microporous cellulose ester membrane in a planar counter-flow dialyzer, Yeh and Zhang observed good agreement between experimental results and theoretical predictions from a mass transfer model (Yeh and Chang 2005). This model is based on the classic solutions from Graetz and Lévêque for heat transfer to tube walls for a laminar flow, which can be extrapolated to mass transfer because of the identical forms of the convection-diffusion equation (Kirtland 2010). As an idealization, it is assumed that the solute is consumed at the channel wall, i.e., the membrane in a dialyzer, that the time scale for solute diffusion is longer than the time scale of axial convection ( flow rate), and that there is no radial convection. This enables calculation of the diffusive solute flux to this boundary wall and its scaling with the flow rate as a function of the distance from the feed channel inlet, which takes the form of a $1 / 3$ power law for the parabolic velocity profile of a fully developed laminar flow (Kirtland 2010; Haase et al. 2015). Here, we follow the model derived by Yeh et al. (Yeh and Hsu 1999; Yeh and Chang 2005), where the total mass transfer rate $M(\mathrm{~mol} / \mathrm{s})$ in a planar counter-flow dialyzer is given by Eq. (2). This analysis also assumes: steady-state diffusion, uniform solute concentration over the cross section of flow, a constant overall mass transfer coefficient, constant flow rates, constant physical properties of the fluid and no chemical reactions (Yeh and Chang 2005).

$M=\frac{\left(C_{\mathrm{f}, \text { in }}-C_{\mathrm{w}, \text { in }}\right)\left(1-\mathrm{e}^{-K S\left[\frac{1}{Q_{\mathrm{f}}}-\frac{1}{Q_{\mathrm{w}}}\right]}\right)}{\left(\frac{1}{Q_{\mathrm{f}}}\right)-\left(\frac{1}{Q_{\mathrm{w}}}\right) \mathrm{e}^{-K S\left[\frac{1}{Q_{\mathrm{f}}}-\frac{1}{Q_{\mathrm{w}}}\right]}}$

Here, $C_{\mathrm{f}, \mathrm{in}}$ is the solute concentration $\left(\mathrm{mol} / \mathrm{m}^{3}\right)$ of the predialysis feed solution and $C_{\mathrm{w}, \mathrm{in}}$ of the pre-dialysis water flow (i.e., no solute). $Q_{\mathrm{f}}$ and $Q_{\mathrm{w}}$ are the flow rates $\left(\mathrm{m}^{3} / \mathrm{s}\right)$ of the feed and the water phase, respectively, and $S$ is the feedexposed mass transfer area of the membrane sheet $\left(\mathrm{m}^{2}\right)$. The overall mass transfer coefficient $K(\mathrm{~m} / \mathrm{s})$ is:

$\frac{1}{K}=\frac{1}{k_{\mathrm{f}}}+\frac{1}{k_{\mathrm{m}}}+\frac{1}{k_{\mathrm{w}}}$,

where $k_{\mathrm{f}}$ and $k_{\mathrm{w}}$ are the solute mass transfer coefficients for the feed and the water phase, respectively, and $k_{\mathrm{m}}$ for the membrane, derived as:

$k_{\mathrm{f}}=0.816\left(\frac{6 Q_{\mathrm{f}} D_{\mathrm{f}}^{2}}{W_{\mathrm{f}} H_{\mathrm{f}}^{2} L_{\mathrm{f}}}\right)^{\frac{1}{3}}$,

$k_{\mathrm{w}}=0.816\left(\frac{6 Q_{\mathrm{w}} D_{\mathrm{w}}^{2}}{W_{\mathrm{w}} H_{\mathrm{w}}^{2} L_{\mathrm{w}}}\right)^{\frac{1}{3}}$,

$k_{\mathrm{m}}=\frac{D_{\mathrm{m}} \varepsilon}{\tau t}$.

Here, $W, H$ and $L$ are the width, height and length (m) of the feed or the water channel, and $D$ is the diffusion coefficient $\left(\mathrm{m}^{2} / \mathrm{s}\right)$ of the solute in each phase, as indicated by the subscript. The structural parameters of the membrane are included as membrane porosity $\varepsilon$, tortuosity $\tau$ and thickness $t$ (m) (Yeh 2008).

The absolute salt ion mass (mol) transferred from the feed to the water phase is obtained from the total mass transfer rate $M(\mathrm{~mol} / \mathrm{s})$ by considering the membrane contact time (s) of a segment of the laminar feed stream at a given flow rate. For example, given the $\sim 25 \mu \mathrm{L}$ volume of the feed channel, a feed flow of $10 \mu \mathrm{L} / \mathrm{min}$ corresponds to a dialyzer 
flow-through time of $\sim 2.5 \min (150 \mathrm{~s})$. The theoretical postdialysis salt concentration of the feed solution can then be calculated by subtracting the theoretical amount of salt transferred to the water phase from the pre-dialysis amount of salt in the feed channel volume.

\section{Results and discussion}

\subsection{Membrane characterization}

Membranes with a nominal MWCO of 1-10 kDa are permeable to salt ions but reject most protein species. Cross sections of regenerated cellulose UF membranes were visualized with electron microscopy, revealing the typical ultrastructure of membranes synthesized by phase inversion (Cuperus and Smolders 1991). Figure 1a shows a UF membrane of $\sim 20 \mu \mathrm{m}$ thickness on top of its $\sim 180 \mu \mathrm{m}$ thick microporous support sheet. The asymmetric structure of the UF membrane is apparent at higher magnification (Fig. 1b), showing a dense skin layer of $\sim 2 \mu \mathrm{m}$ thickness on top of $\mathrm{a} \sim 20 \mu \mathrm{m}$ thick sub-layer with a $\sim 200 \mathrm{~nm}$ diameter pore structure. The pores in the sample-facing skin layer, which determine the separation performance, will have a diameter of $\sim 2 \mathrm{~nm}$ (Cuperus and Smolders 1991; Romero et al. 2013).

The porosity of the membranes was estimated by electrical measurements. The $I-V$ relationship for the UF membranes and for the reference track-etch membrane is depicted in Fig. 1c. The conductance of 1, 5 and $10 \mathrm{kDa}$ polyethersulfone membranes was 104,116 and $173 \mu \mathrm{S}$, respectively; while the conductance of the $50 \mathrm{~nm}$ pore diameter track-etch membrane was $184 \mu \mathrm{S}$. Estimating the UF and the track-etch membrane thickness as $20 \mu \mathrm{m}$ and taking the porosity of the reference track-etch membrane, as specified by the supplier, as $1.18 \%$, the porosity of the 1,5 and $10 \mathrm{kDa}$ membranes can be approximated as, respectively, $0.67,0.74$ and $1.11 \%$. The conductance measured for a $5 \mathrm{kDa}$ regenerated cellulose membrane was $112 \mu \mathrm{S}$, corresponding to a porosity of $0.72 \%$. These values agree with previously reported values for UF membrane porosities, which range from 0.1 to $10 \%$ but are typically around 0.5\% (Cuperus and Smolders 1991).

\subsection{Effect of water flow rates on static sample dialysis}

Regenerated cellulose membranes of $5 \mathrm{kDa}$ MWCO were clamped between two acrylic plates with spiral microfluidic channels defined by laser machining, matching the circular outline of the membrane (Fig. 2a). Tight contact between the top and bottom plate ensured a good seal with the polymer membrane, as previously demonstrated for other microfluidic dialyzers (Kurita et al. 2006; Sheng and Bowser 2012; Perozziello et al. 2015). Initial desalting experiments were performed with a static feed solution of $150 \mathrm{mM} \mathrm{NaCl}$ and $2 \mathrm{mg} / \mathrm{mL}$ fluorescently labeled BSA (to visually verify BSA retention in the feed channel) with co-lyophilized buffer ions
Fig. 1 a Scanning electron microscopy image of a cross section of a regenerated cellulose (RC) ultrafiltration membrane on top of its microporous support sheet. b Cross section of the membrane itself, with the dense skin layer visible in the top left corner and the sub-layer, partially covered by debris, in the center of the image. c Current-voltage relationships for $\mathrm{RC}$ and polyethersulfone (PES) ultrafiltration membranes of various MWCO specifications and a reference polycarbonate (PC) track-etch membrane of known porosity
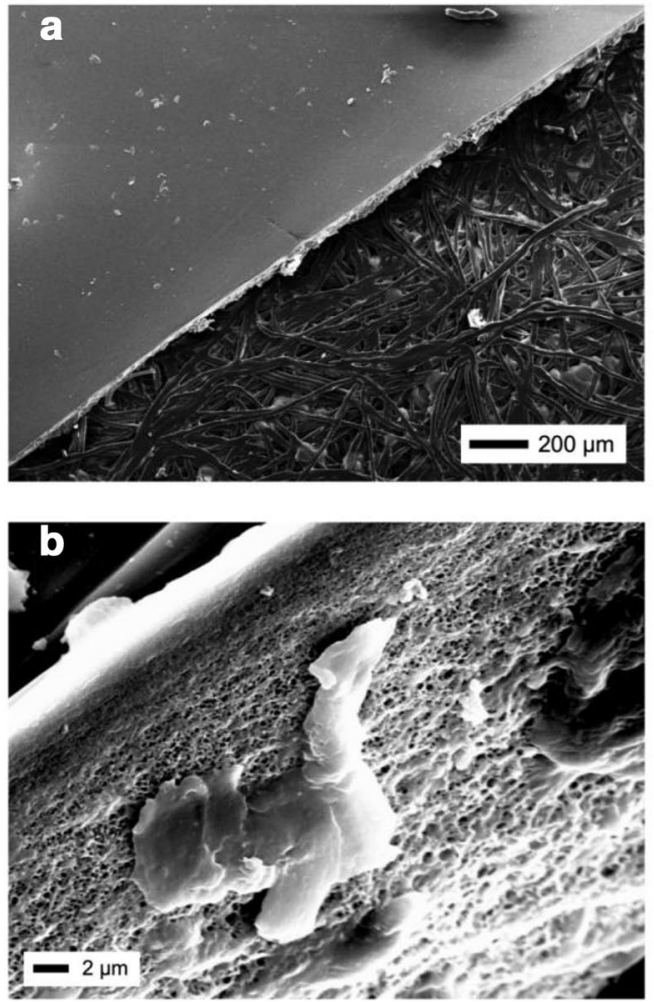

c

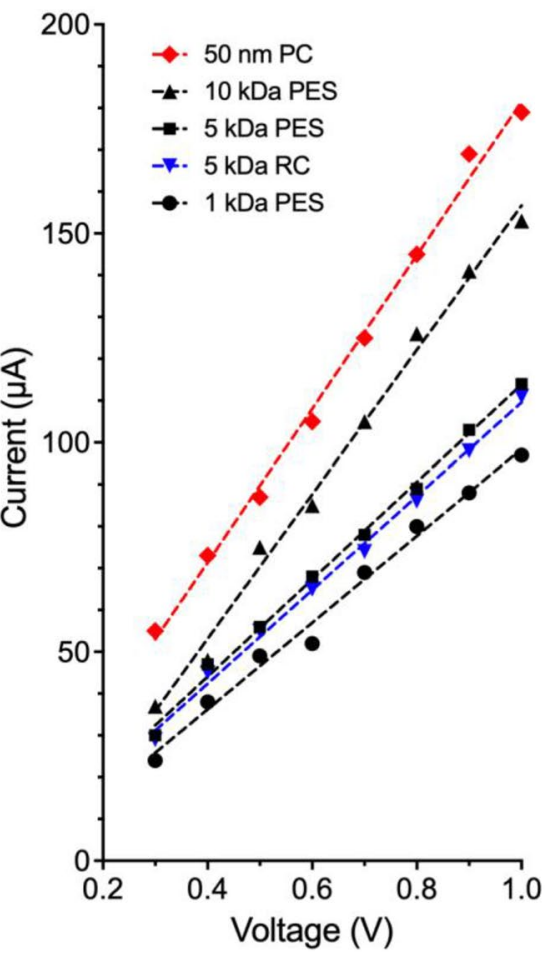


a

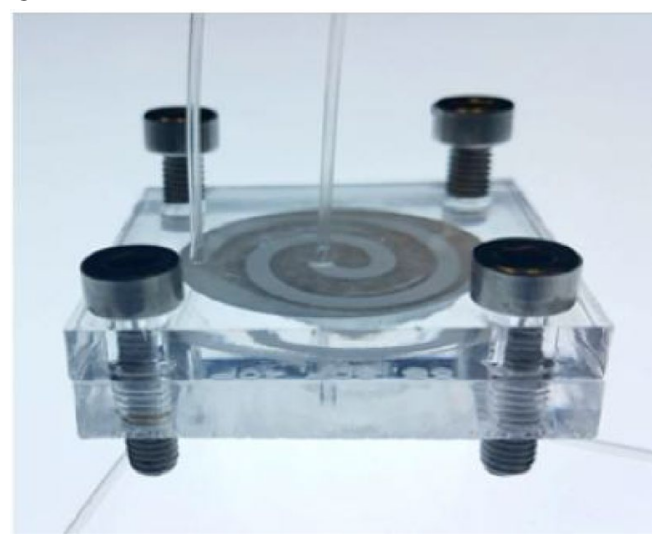

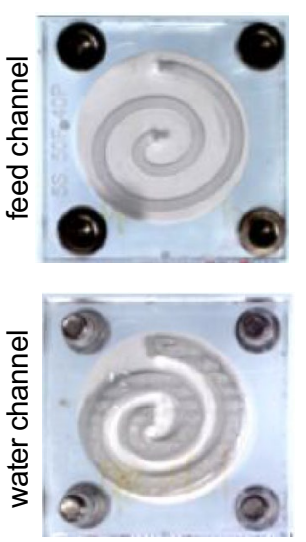

b

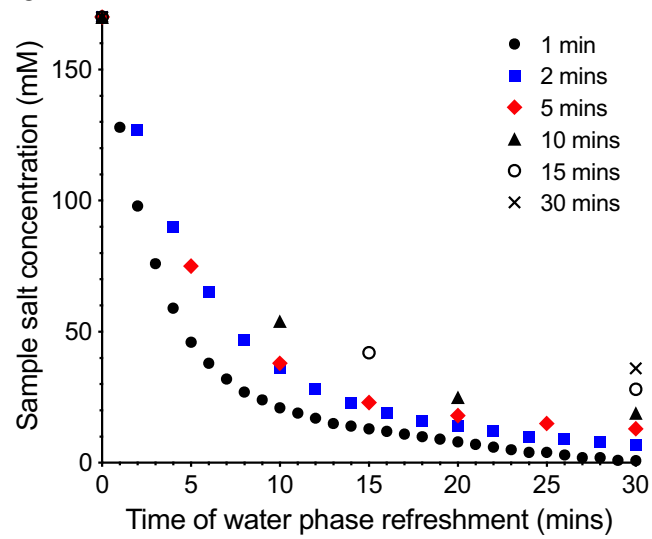

Fig. 2 a Counter-flow microfluidic dialyzer with an ultrafiltration membrane sealed between $3 \times 3 \mathrm{~cm}$ acrylic plates with lasermachined feed and water channels in a spiral geometry. b Residual salt concentration of a static sample phase as a function of periodic refreshment, at different time intervals, of a static water phase
(170 mM total salt) and a static dialysate phase of deionized water, which was refreshed at different time intervals. After removal from the dialyzer, the conductivity of the dialysate was measured to determine the amount of membrane-permeated salt ions, enabling estimation of the remaining salt concentration in the feed chamber after each refreshment step.

Figure $2 b$ shows that, in general, a higher frequency of water refreshment (i.e., shorter water phase resident time in the dialyzer) results in a lower residual salt concentration in the feed chamber. For example, after $15 \mathrm{~min}$, when the water phase has been refreshed one, two, three or 15 times, the residual salt amounts to $25 \%, 19 \%, 14 \%$ or $8 \%$, respectively, of the original concentration. This corresponds to a molar solute flux across the membrane of $2-3 \times 10^{-5} \mathrm{~mol} / \mathrm{m}^{2} / \mathrm{s}$. Figure $2 \mathrm{~b}$ also illustrates that the initial water refreshment steps yield the most pronounced reduction in salt concentration of the static feed sample. This is expected because the feed/dialysate salt concentration gradient, the driving force for dialysis, is largest at the initial stage of desalting. When the water phase is refreshed every minute, the salt concentration in the feed sample is reduced to approximately $1 \mathrm{mM}$ after $30 \mathrm{~min}$. By varying the amount of time that the water phase is in contact with the static salt sample and the rate at which the water is refreshed, the extent of desalting can be modulated.

\subsection{Effect of flow rates on counter-flow dynamic dialysis}

A continuously flowing feed stream enables higher sample throughput and facilitates dialysis quantification by in-line conductivity measurements of the post-dialysis retentate phase, i.e., the desalted feed. Deionized water continuously flowing in the opposite direction enables constant refreshment of the permeate ('water') phase, maintaining maximal concentration gradients. To quantify the separation performance of the counter-flow dialyzer, we determined the post-dialysis retentate salt concentration for a wide range of flow conditions: $10-600 \mu \mathrm{L} / \mathrm{min}(0.016-0.95 \mathrm{~mm} / \mathrm{s}$ linear velocity) for the water phase and $0.5-50 \mu \mathrm{L} / \mathrm{min}$ $(0.023-2.3 \mathrm{~mm} / \mathrm{s})$ for the feed phase. Data points represent single measurements. For a number of flow combinations, repeat experiments were performed, indicating a variation of a few mM.

Figure $3 \mathrm{a}$ depicts the percentage of salt retained at the feed side of the $5 \mathrm{kDa}$ membrane at a fast water flow of $600 \mu \mathrm{L} / \mathrm{min}$, which approximates the highest frequency of water refreshment in the quasi-dynamic dialyzer operation (Fig. 2b), and at a slow flow of $10 \mu \mathrm{L} / \mathrm{min}$. For the $600 \mu \mathrm{L} /$ min water flow, it can be seen that a slow feed flow of 0.5 $\mu \mathrm{L} / \mathrm{min}$ leads to almost complete desalting $(0.2 \mathrm{mM}$ retentate salt, i.e., $0.1 \%$ of salt retained) and a fast feed flow of $50 \mu \mathrm{L} /$ min to minimal desalting ( $92 \%$ of salt retained). Intermediate feed flow rates result in intermediate salt transfer, suggesting that any particular desalting efficiency can be obtained with the appropriate feed flow rate. A typical syringe pump can set the flow rate in increments of $0.01 \mu \mathrm{L} / \mathrm{min}$.

Figure $3 \mathrm{a}$ also shows that the water flow rate modulates the dialysis performance. At a slow water flow of $10 \mu \mathrm{L} /$ min, there is more salt retained in the sample for all feed flow rates. Salt transfer over the membrane is minimal for feed flows above $20 \mu \mathrm{L} / \mathrm{min}(0.93 \mathrm{~mm} / \mathrm{s})$ but the lowest flow rate of $0.5 \mu \mathrm{L} / \mathrm{min}$ results in efficient desalting $(0.6 \%$ of salt retained). Figure $3 \mathrm{~b}$ shows the percentage of salt retained in the feed stream at the lowest sample flow rates of 0.5 and 1.0 $\mu \mathrm{L} / \mathrm{min}$, where salt transfer is extremely efficient. At $0.5 \mu \mathrm{L} /$ min, over $99 \%$ of the salt ions diffuse over the membrane to the water phase for the entire water flow range of $10-600 \mu \mathrm{L} /$ 

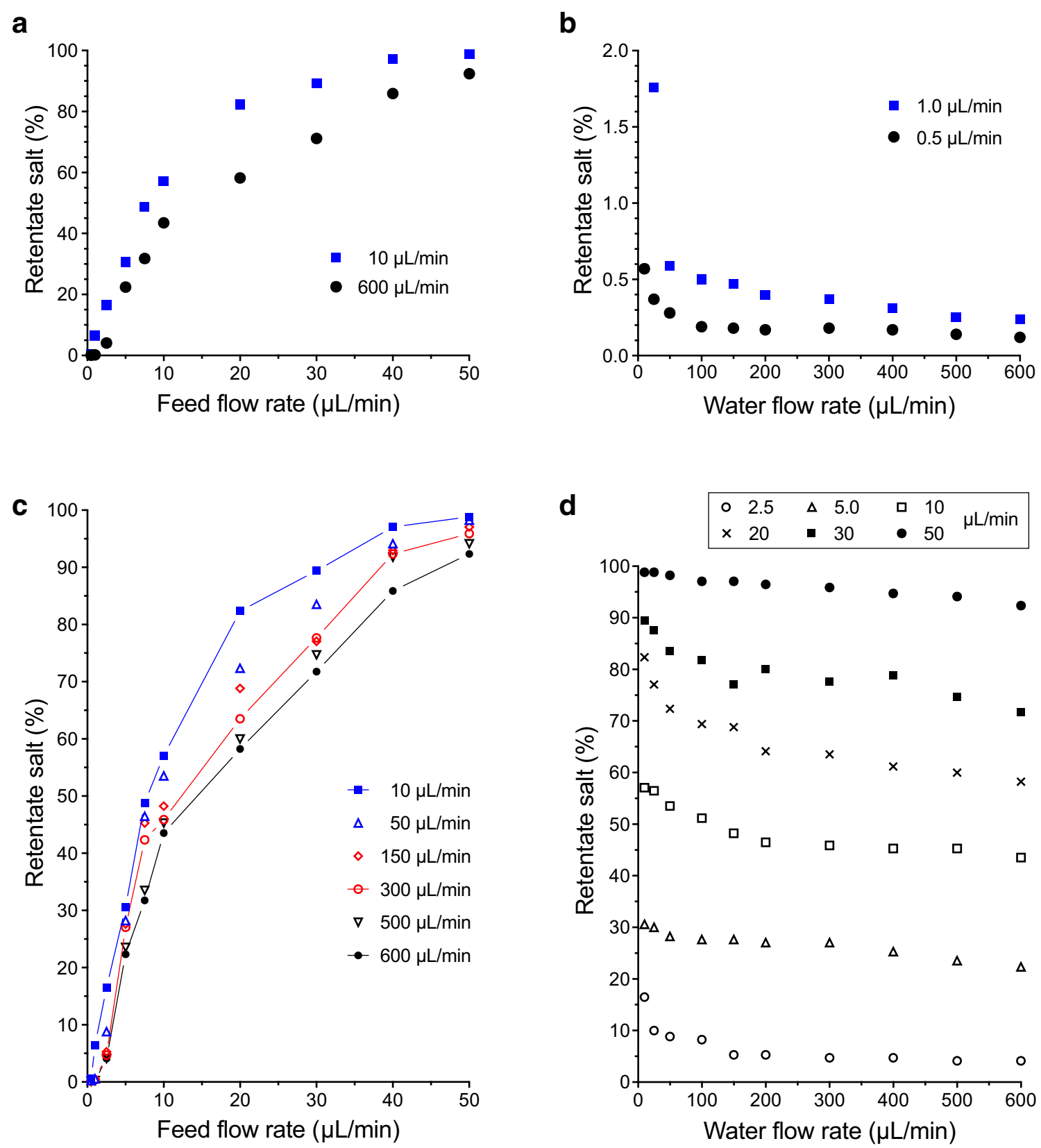

Fig. 3 Retentate salt concentration after dynamic counter-flow dialysis with a $5 \mathrm{kDa}$ MWCO regenerated cellulose ultrafiltration membrane at various feed and water flow rate combinations, as a percentage of the initial salt concentration. a Feed flows from 0.5 to $50 \mu \mathrm{L} /$ min at water flow rates of 10 and $600 \mu \mathrm{L} / \mathrm{min}$. b Water flows from 10 to $600 \mu \mathrm{L} / \mathrm{min}$ at feed flow rates of 0.5 and $1.0 \mu \mathrm{L} / \mathrm{min}$. c Feed flows from 0.5 to $50 \mu \mathrm{L} / \mathrm{min}$ at water flows of $10,20,50,150,300$,

$\min$. At $1.0 \mu \mathrm{L} / \mathrm{min}(0.046 \mathrm{~mm} / \mathrm{s})$ feed flow this necessitates water flow rates from $50 \mu \mathrm{L} / \mathrm{min}(0.079 \mathrm{~mm} / \mathrm{s})$, with a 10 and $25 \mu \mathrm{L} / \mathrm{min}$ water flow giving $6.5 \%$ (outside the frame of Fig. $3 \mathrm{~b})$ and $1.8 \%$ retentate salt, respectively.

For water flow rates of $10,50,150,300,500$ and 600 $\mu \mathrm{L} / \mathrm{min}$, the retentate residual salt percentage is shown as
500 and $600 \mu \mathrm{L} / \mathrm{min}$. Lines connecting data points are a guide to the eye. d Water flows from 10 to $600 \mu \mathrm{L} / \mathrm{min}$ at six different feed flow rates between 2.5 and $50 \mu \mathrm{L} / \mathrm{min}$. The sample is $150 \mathrm{mM} \mathrm{NaCl}$ with $2 \mathrm{mg} / \mathrm{mL}$ fluorescently labeled BSA with co-lyophilized buffer ions: $170 \mathrm{mM}$ total salt. Note that volumetric feed and water flow rates correspond to different flow velocities because of the larger volume of the water channel

a function of the feed flow rate in Fig. 3c. The residual salt fraction is plotted as a function of the water flow rate in Fig. 3d. For all water flow rates, the salt dialysis efficiency clearly depends on the sample flow rate; a slower sample flow results in a lower retentate salt concentration (Fig. 3c), i.e., more efficient salt transfer over the membrane. At higher 
sample flow rates, the efficiency is reduced because the residence time of the sample in the feed channel becomes shorter, as previously observed for other microdialyzers (Jakubowski et al. 2005; Sheng and Bowser 2012). A short diffusion distance to the membrane is crucial for efficient dialysis (Roelofs et al. 2015). The Brownian motion diffusion coefficient of $\mathrm{NaCl}$ at $\sim 1-150 \mathrm{mM}$ concentration at $25{ }^{\circ} \mathrm{C}$ is $\sim 1.5 \times 10^{-5} \mathrm{~cm}^{2} / \mathrm{s}$ (Robinson and Stokes 2002), which corresponds to a net ion displacement of $\sim 400 \mu \mathrm{m} /$ min; while the height of the feed channel is $200 \mu \mathrm{m}$. This implies that for optimal salt transfer efficiencies, the contact time of the sample stream with the membrane should be at least $30 \mathrm{~s}$. Given the $75 \mathrm{~mm}$ length of the feed channel, this implies a linear velocity of at most $2.5 \mathrm{~mm} / \mathrm{s}$, i.e., a sample flow rate of at most $2.5 \mu \mathrm{L} / \mathrm{min}$.

At a particular sample flow rate, a faster water flow, in the opposite direction, generally improves salt transfer, but this modulation by the water counter-flow is modest (Fig. 3d). For example, at a sample flow of $10 \mu \mathrm{L} / \mathrm{min}(0.46 \mathrm{~mm} / \mathrm{s})$, for the slowest water flow of $10 \mu \mathrm{L} / \mathrm{min}(0.02 \mathrm{~mm} / \mathrm{s}) \sim 57 \%$ of salt is retained in the feed; while this is $\sim 44 \%$ for the highest flow rate of $600 \mu \mathrm{L} / \mathrm{min}(0.95 \mathrm{~mm} / \mathrm{s})$. For several feed flow rates, the desalting efficiency improves most when the water counter-flow is increased from 10 to $\sim 150 \mu \mathrm{L} / \mathrm{min}$ (Fig. 3d). This is due to the relative residence times of the feed and the water phase (Yeh and Hsu 1999); at the lowest water flow rates, the linear velocity of the water stream is considerably smaller than the velocity of the feed stream. For example, the $10 \mu \mathrm{L} / \mathrm{min}$ feed velocity is nearly 30 times faster than the $0.016 \mathrm{~mm} / \mathrm{s}$ velocity of the $10 \mu \mathrm{L} / \mathrm{min}$ water flow. Under these conditions, the water counter-flow is, hence, somewhat static with respect to the feed flow, which diminishes the salt concentration gradient over the membrane. Increasing the water flow velocity, up to $0.32 \mathrm{~mm} / \mathrm{s}(200 \mu \mathrm{L} / \mathrm{min})$, can, thus, noticeably enhance the dialysis efficiency.

\subsection{Mass transfer analysis}

The mass transfer over the membrane in the counter-flow dialyzer was modeled as described in Sect. 2.4. Mass transfer coefficients for the feed and the water phase were calculated for flow rates up to 50 and $600 \mu \mathrm{L} / \mathrm{min}$, respectively. For $k_{\mathrm{m}}$ we used the experimentally determined membrane porosity of $0.72 \%$ and a membrane skin layer thickness of $2 \mu \mathrm{m}$ (Sect. 3.1). Ultrafiltration membranes have a 3D pore network; hence, the tortuosity was approximated as 2 (Kokubo and Sakai 1998; Bakeri et al. 2015). The diffusion coefficient of the salt ions inside the pores was assumed to be identical to bulk solution, i.e., $1.5 \times 10^{-5} \mathrm{~cm}^{2} / \mathrm{s}$ (Robinson and Stokes 2002), giving a $k_{\mathrm{m}}$ of $2.7 \times 10^{-6} \mathrm{~m} / \mathrm{s}$. The overall mass transfer coefficient $K(\mathrm{~m} / \mathrm{s})$ for transport from the feed to the water phase was then obtained according to Eq. (3), and subsequently the overall mass transfer rate $M(\mathrm{~mol} / \mathrm{s})$ was derived according to Eq. (2).

These theoretical values for $K$ and $M$ are shown in Fig. 4a, $\mathrm{b}$, respectively, for the experimental water flow rates as well as for smaller values of 1 and $5 \mu \mathrm{L} / \mathrm{min}$, as a function of the sample flow rate $(0.5-50 \mu \mathrm{L} / \mathrm{min}) . K$ varies between $\sim 0.3$ and $1.2 \mu \mathrm{m} / \mathrm{s}$, increasing with faster sample and water flows. The steep increase in $M$ for sample flow rates up to $\sim 10$ $\mu \mathrm{L} / \mathrm{min}$ represents the transition from a static to an effective dynamic feed phase, substantially increasing the salt concentration gradient over the membrane. Also the mass transfer increase with higher water flow rates, from $\sim 3$ to $25 \mathrm{nmol} / \mathrm{s}$, can be related to a larger concentration gradient. However, the effect of operating the counter-flow dialyzer at a water flow beyond $300 \mu \mathrm{L} / \mathrm{min}$ is relatively small; the salt gradient is not expected to increase significantly in this fast flow regime.

For selected feed flow rates, the modeled post-dialysis salt concentration of the feed phase, obtained from $M$ as described in Sect. 2.4, was compared with the experimentally determined concentrations. Figure $4 \mathrm{c}$ shows that the theoretical and experimental values are well matched, generally deviating by at most $\sim 10 \%$ retentate salt concentration. Such good agreement between diffusion theory and experiment was also observed for counter-flow dialysis of urea over a cellulose ester membrane (Yeh and Chang 2005), whose model was applied here, and of fluorescein over a track-etched polycarbonate membrane (Sheng and Bowser 2012), as well as for co-flow dialysis of salt ions over a nanoporous alumina membrane (Tibavinsky et al. 2015). The models of the latter two studies likewise assume advection-dominated solute transport along the feed channel and diffusion-dominated transport over the membrane (i.e., minimal transmembrane water flow and hence no or negligible solute advection over the membrane), with feed flow rates determining the membrane contact time and water flow rates modulating the salt concentration gradient over the membrane.

\subsection{Dialysis performance at high protein concentration}

Counter-flow salt dialysis was also performed with a sample of $170 \mathrm{mM}$ salt and $50 \mathrm{mg} / \mathrm{mL}(0.75 \mathrm{mM})$ BSA $(2 \mathrm{mg} / \mathrm{mL}$ fluo-BSA and $48 \mathrm{mg} / \mathrm{mL}$ non-labeled BSA), approximating the total protein concentration in human blood serum, using membranes with different MWCO values. At the lowest sample flow rate of $0.5 \mu \mathrm{L} / \mathrm{min}$, the post-dialysis retentate conductivity was found to correspond to $1 \%$ of the input salt concentration for 5 and $10 \mathrm{kDa}$ MWCO membranes and to $2 \%$ for a $1 \mathrm{kDa}$ MWCO membrane, for all investigated water flow rates (in the range of $10-750 \mu \mathrm{L} / \mathrm{min}$, data not shown). For the three membranes, Fig. 5 depicts the 


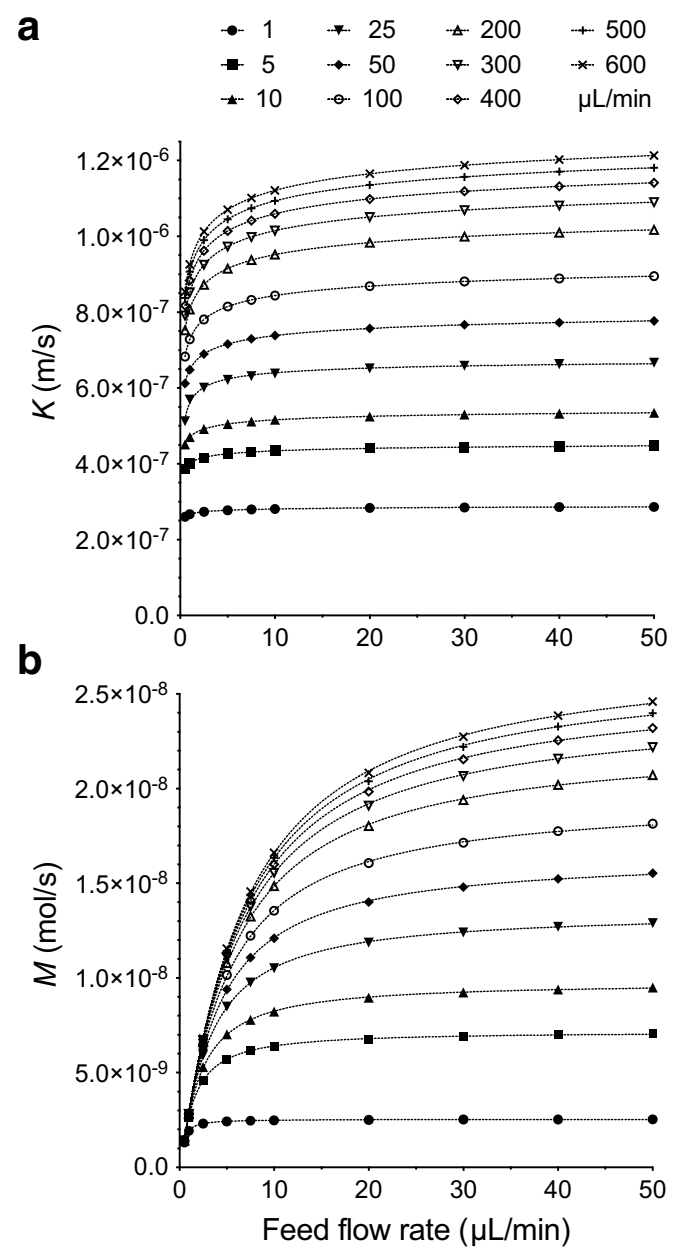

Fig. 4 a Theoretical overall mass transfer coefficient $K$ and $\mathbf{b}$ theoretical mass transfer rate $M$ for the counter-flow dialyzer for feed flows from 0 to $50 \mu \mathrm{L} / \mathrm{min}$ at discrete water flow rates between 1 and 600 $\mu \mathrm{L} / \mathrm{min}$. c Comparison between the theoretically predicted (connected
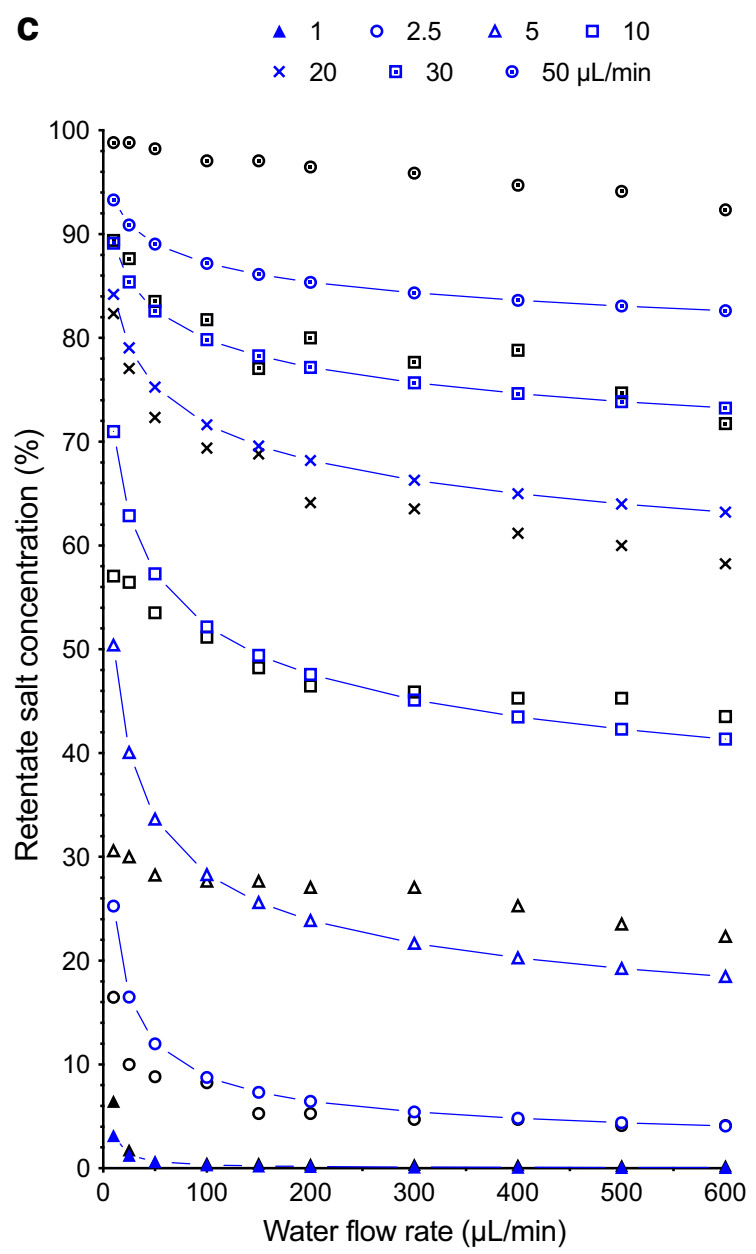

blue markers) and the experimentally obtained (black markers) retentate salt concentration for feed flow rates of 1, 2.5, 5, 10, 20, 30 and $50 \mu \mathrm{L} / \mathrm{min}$

retentate salt concentration for sample flow rates from 0.5 to $50 \mu \mathrm{L} / \mathrm{min}$ at a fast water flow. As previously observed for a model sample with $2 \mathrm{mg} / \mathrm{mL}$ BSA and a dialysis cell with a $5 \mathrm{kDa}$ MWCO membrane (Fig. 3a), the dialysis efficiency depends on the feed flow rate. At feed flows from $10 \mu \mathrm{L} / \mathrm{min}$, compared to the $5 \mathrm{kDa}$ MWCO membrane, the $10 \mathrm{kDa}$ membrane results in $\sim 10 \%$ more salt transfer to the water phase, with respect to the input salt concentration, and the $1 \mathrm{kDa}$ membrane in $\sim 10 \%$ less. Qualitatively, this trend reflects the different porosities of these membranes (Sect. 3.1).

For feed flow rates up to $30 \mu \mathrm{L} / \mathrm{min}$, the residual salt percentage obtained with a $5 \mathrm{kDa}$ MWCO membrane is $~ 15 \%$ higher than for the model sample with $2 \mathrm{mg} / \mathrm{mL}$ BSA (Fig. 3a), indicating reduced mass transfer due to membrane fouling associated with the high protein concentration of $50 \mathrm{mg} / \mathrm{mL}$. It should be noted that BSA has been employed as a model foulant in studies of ultrafiltration cells (Wang and Tang 2011), which are prone to fouling because of the 
applied transmembrane pressure. To assess any further decrease in separation performance over time, a counter-flow dialysis cell with a $5 \mathrm{kDa}$ MWCO membrane was operated continuously for $16 \mathrm{~h}$ with a feed and water flow rate of 0.5 and $750 \mu \mathrm{L} / \mathrm{min}$, respectively. Towards the end of this period, the salt concentration of the retentate had increased by just $\sim 0.1 \mathrm{mM}$ with respect to the start of the dialyzer operation. Hence, any membrane fouling does not become more severe by prolonged counter-flow dialysis, where the feed flowing parallel to the membrane surface is expected to minimize membrane fouling because of shear stress (Casey et al. 2011).

\section{Conclusions}

We characterized the dialysis performance of a microfluidic counter-flow dialyzer with a $\sim 25 \mu \mathrm{L}$ feed and a $800 \mu \mathrm{L}$ dialysate channel of $75 \mathrm{~mm}$ length, separated by a commercial $5 \mathrm{kDa}$ MWCO cellulose membrane. The feed and dialysis flow rates determine the retentate salt concentration, indicating that the separation efficiency can be precisely controlled by setting these flow rates. Specifically, with a feed flow between 0.5 and $50 \mu \mathrm{L} / \mathrm{min}(0.023$ and $2.3 \mathrm{~mm} / \mathrm{s})$, the retentate salt concentration varies from $\sim 0.1$ to $~ 99 \%$ of the input sample of $170 \mathrm{mM}$ salt. For a given feed flow rate, a faster water flow also improves salt transfer by increasing the concentration gradient over the membrane. But because the counter-flow configuration is very efficient in realizing a steep gradient, this effect is relatively modest (up to a $20 \%$ improvement). Mass transfer modeling shows that a faster sample flow actually also results in a higher mass transfer rate (up to $\sim 5-10 \mathrm{nmol} / \mathrm{s}$ ) because in a counter-flow geometry, this again increases the concentration gradient. However, the dominant effect of a faster sample flow is a shorter dialyzer flow-through time and hence a reduced membrane contact time of the feed phase, resulting in less mass transfer and hence less efficient desalting of the feed flow.

The time required for desalting a sample by counterflow dialysis could be reduced by implementing a feed channel shallower than $200 \mu \mathrm{m}$, reducing the diffusion distance to the membrane; while the volume throughput of the dialyzer could be maintained with a wider feed channel or multiple parallel channels. However, sample volumes of $\sim 100 \mu \mathrm{L}$ are sufficient for many analytical techniques and can be dialyzed in a reasonable timeframe with the present geometry. For longer flow-through dialysis times (i.e., larger sample volumes), the desalting performance did not significantly deteriorate with $50 \mathrm{mg} / \mathrm{mL}$ of the model foulant protein BSA, which is a major advantage of dialysis with respect to a pressure-driven separation process such as ultrafiltration (Shi et al. 2014). Salt transfer efficiency could also be increased, and process time decreased, by employing a membrane of higher porosity. For the 1-10 kDa MWCO regime which enables sample retention (membrane rejection) of biomarker proteins, planar membranes as supplied for ultrafiltration applications are of very low porosity, but the synthesis of nanoporous membranes of reduced thickness and higher porosity is an active research area (DesOrmeaux et al. 2014; Mireles and Gaborski 2017; Wang et al. 2017; Wu et al. 2018).

Generally, our rapid prototyping approach of clamping membranes between rigid laser-machined acrylic plates enables implementation of tuneable dynamic dialysis for any planar membrane material and any diffusion-driven separation process. Specifically, we anticipate that tuneable desalting will benefit molecular analysis methods such as field effect transistor biosensing (Noor and Krull 2014; Kaisti 2017; Lowe et al. 2017), where a reduced salt concentration is required for sensing but excessive desalting can disrupt biomolecular structure and interactions.

Acknowledgements This work was supported by the UK Engineering and Physical Sciences Research Council (EP/K502327/1). P. C. K. was awarded a studentship from the School of Electronics and Computer Science. We are grateful to Sumit Kalsi for SEM imaging.

Data availability All data supporting this study are openly available from the University of Southampton repository at https://doi. org/10.5258/SOTON/D0733.

Open Access This article is distributed under the terms of the Creative Commons Attribution 4.0 International License (http://creativeco mmons.org/licenses/by/4.0/), which permits unrestricted use, distribution, and reproduction in any medium, provided you give appropriate credit to the original author(s) and the source, provide a link to the Creative Commons license, and indicate if changes were made.

\section{References}

Bakeri G, Naeimifard S, Matsuura T, Ismail AF (2015) A porous polyethersulfone hollow fiber membrane in a gas humidification process. RSC Adv 5:14448-14457. https://doi.org/10.1039/ C4RA14180F

Canarelli S, Fisch I, Freitag R (2002) On-line microdialysis of proteins with high-salt buffers for direct coupling of electrospray ionization mass spectrometry and liquid chromatography. $\mathrm{J}$ Chromatogr A 948:139-149. https://doi.org/10.1016/S0021 -9673(01)01344-9

Casey C, Gallos T, Alekseev Y, Ayturk E, Pearl S (2011) Protein concentration with single-pass tangential flow filtration (SPTFF). J Membr Sci 384:82-88. https://doi.org/10.1016/j. memsci.2011.09.004

Chen X, Shen J, Hu Z, Huo X (2016) Manufacturing methods and applications of membranes in microfluidics. Biomed Microdevices 18:104. https://doi.org/10.1007/s10544-016-0130-7

Cuperus FP, Smolders CA (1991) Characterization of UF membranes: membrane characteristics and characterization techniques. Adv Colloid Interface Sci 34:135-173. https://doi. org/10.1016/0001-8686(91)80049-P 
de Jong J, Lammertink RGH, Wessling M (2006) Membranes and microfluidics: a review. Lab Chip 6:1125-1139. https://doi. org/10.1039/b603275c

DesOrmeaux JPS, Winans JD, Wayson SE, Gaborski TR, Khire TS, Striemer CC, McGrath JL (2014) Nanoporous silicon nitride membranes fabricated from porous nanocrystalline silicon templates. Nanoscale 6:10798-10805. https://doi.org/10.1039/ C4NR03070B

Garg R, Kumar V, Kumar D, Chakarvarti SK (2012) Electrical transport through micro porous track etch membranes of same porosity. Mod Phys Lett B 26:1250209. https://doi.org/10.1142/ S0217984912502090

Garg R, Kumar V, Kumar D, Chakarvarti SK (2014) Electrical conduction studies through micro porous track etch membranes of equal pore density and porosity. ISST J Appl Phys 5:101-105

Haase AS, Chapman SJ, Tsai PA, Lohse D, Lammertink RGH (2015) The Graetz-Nusselt problem extended to continuum flows with finite slip. J Fluid Mech 764:R3. https://doi.org/10.1017/jfm.2014.733

Han S, Hwang DK (2018) No more bonding, no more clamping, magnetically assisted membrane integration in microfluidic devices. Microfluid Nanofluid 22:107. https://doi.org/10.1007/s10404-018-2127-4

Jakubowski JA, Hatcher NG, Sweedler JV (2005) Online microdialysisdynamic nanoelectrospray ionization-mass spectrometry for monitoring neuropeptide secretion. J Mass Spectrom 40:924-931. https ://doi.org/10.1002/jms.869

Kaisti M (2017) Detection principles of biological and chemical FET sensors. Biosens Bioelectron 98:437-448. https://doi.org/10.1016/j. bios.2017.07.010

Kazemi AS, Kawka K, Latulippe DR (2016) Optimization of biomolecule separation by combining microscale filtration and design-ofexperiment methods. Biotechnol Bioeng 113:2131-2139. https:// doi.org/10.1002/bit.25975

Kirtland JD (2010) Interfacial mass transfer in microfluidic systems: existence and persistence of the modified Grætz behavior. Ph.D. thesis, Cornell University

Kokubo K, Sakai K (1998) Evaluation of dialysis membranes using a tortuous pore model. AIChE J 44:2607-2619. https://doi.org/10.1002/ aic. 690441204

Kornreich M, Heymann M, Fraden S, Beck R (2014) Cross polarization compatible dialysis chip. Lab Chip 14:3700-3704. https://doi. org/10.1039/c4lc00600c

Kumar S, Chakarvarti SK (2008) Measurement of average etched pore radius in ion track membranes through conductometric technique. Mod Phys Lett B 22:2993-2998. https://doi.org/10.1142/S0217 984908017527

Kurita R, Yabumoto N, Niwa O (2006) Miniaturized one-chip electrochemical sensing device integrated with a dialysis membrane and double thin-layer flow channels for measuring blood samples. Biosens Bioelectron 21:1649-1653. https://doi.org/10.1016/j. bios.2005.07.016

Kwak R, Guan G, Peng WK, Han J (2013) Microscale electrodialysis: concentration profiling and vortex visualization. Desalination 308:138-146. https://doi.org/10.1016/j.desal.2012.07.017

Liu C, Verma SS (1999) Direct coupling of ionic high-performance liquid chromatography with electrospray ionization mass spectrometry utilizing a microdialysis junction interface. J Chromatogr A 835:93-104. https://doi.org/10.1016/S0021-9673(98)01069-3

Liu C, Hofstadler SA, Bresson JA, Udseth HR, Tsukuda T, Smith RD, Snyder AP (1998) On-line dual microdialysis with ESI-MS for direct analysis of complex biological samples and microorganism lysates. Anal Chem 70:1797-1801. https://doi.org/10.1021/ac971193k

Lowe BM, Sun K, Zeimpekis I, Skylaris C-K, Green NG (2017) Field-effect sensors-from $\mathrm{pH}$ sensing to biosensing: sensitivity enhancement using streptavidin-biotin as a model system. Analyst 142:4173-4200. https://doi.org/10.1039/c7an00455a
Mireles M, Gaborski TR (2017) Fabrication techniques enabling ultrathin nanostructured membranes for separations. Electrophoresis 38:2374-2388. https://doi.org/10.1002/elps.201700114

Noor MO, Krull UJ (2014) Silicon nanowires as field-effect transducers for biosensor development: a review. Anal Chim Acta 825:1-25. https://doi.org/10.1016/j.aca.2014.03.016

Perozziello G, Candeloro P, Gentile F et al (2015) A microfluidic dialysis device for complex biological mixture SERS analysis. Microelectron Eng 144:37-41. https://doi.org/10.1016/j.mee.2015.02.015

Robinson RA, Stokes RH (2002) Electrolyte solutions: second revised edition. Courier Corporation, Chelmsford

Roelofs SH, van den Berg A, Odijk M (2015) Microfluidic desalination techniques and their potential applications. Lab Chip 15:3428-3438. https://doi.org/10.1039/c5lc00481k

Romero V, Vázquez MI, Benavente J (2013) Study of ionic and diffusive transport through a regenerated cellulose nanoporous membrane. J Membr Sci 433:152-159. https://doi.org/10.1016/j.memsc i. 2013.01 .012

Saxena A, Tripathi BP, Kumar M, Shahi VK (2009) Membrane-based techniques for the separation and purification of proteins: an overview. Adv Colloid Interface Sci 145:1-22. https://doi.org/10.1016/j. cis.2008.07.004

Sheng Y, Bowser MT (2012) Size selective DNA transport through a nanoporous membrane in a PDMS microfluidic device. Analyst 137:1144-1151. https://doi.org/10.1039/c2an15966j

Shi X, Tal G, Hankins NP, Gitis V (2014) Fouling and cleaning of ultrafiltration membranes: a review. J Water Process Eng 1:121-138. https ://doi.org/10.1016/j.jwpe.2014.04.003

Sun L, Duan J, Tao D, Liang Z, Zhang W, Zhang L, Zhang Y (2008) A facile microdialysis interface for on-line desalting and identification of proteins by nano-electrospray ionization mass spectrometry. Rapid Commun Mass Spectrom 22:2391-2397. https://doi. org/10.1002/rcm.3622

Tibavinsky IA, Kottke PA, Fedorov AG (2015) Microfabricated ultrarapid desalting device for nanoelectrospray ionization mass spectrometry. Anal Chem 87:351-356. https://doi.org/10.1021/ac5040083

Timm AC, Shankles PG, Foster CM, Doktycz MJ, Retterer ST (2016) Toward microfluidic reactors for cell-free protein synthesis at the point-of-care. Small 12:810-817. https://doi.org/10.1002/ smll.201502764

van Reis R, Zydney A (2007) Bioprocess membrane technology. J Membr Sci 297:16-50. https://doi.org/10.1016/j.memsci.2007.02.045

Wang Y-N, Tang CY (2011) Fouling of nanofiltration, reverse osmosis, and ultrafiltration membranes by protein mixtures: the role of interfoulant-species interaction. Environ Sci Technol 45:6373-6379. https://doi.org/10.1021/es2013177

Wang J, Zhu J, Zhang Y, Liu J, Van der Bruggen B (2017) Nanoscale tailor-made membranes for precise and rapid molecular sieve separation. Nanoscale 9:2942-2957. https://doi.org/10.1039/C6NR08417F

Wardrip NC, Arnusch CJ (2016) Three-dimensionally printed microfluidic cross-flow system for ultrafiltration/nanofiltration membrane performance testing. J Vis Exp 108:e53556. https://doi. org/10.3791/53556

Wu W, Zhang D, Chen K, Zhou P, Zhao M, Qiao L, Su B (2018) Highly efficient desalting by silica isoporous membrane based microfluidic chip for electrospray ionization mass spectrometry. Anal Chem 90:14395-14401. https://doi.org/10.1021/acs.analchem.8b03934

Xiang F, Lin Y, Wen J, Matson DW, Smith RD (1999) An integrated microfabricated device for dual microdialysis and on-line ESI-ion trap mass spectrometry for analysis of complex biological samples. Anal Chem 71:1485-1490. https://doi.org/10.1021/ac981400w

Xu N, Lin Y, Hofstadler SA, Matson D, Call CJ, Smith RD (1998) A microfabricated dialysis device for sample cleanup in electrospray ionization mass spectrometry. Anal Chem 70:3553-3556. https:// doi.org/10.1021/ac980233x 
Yeh H-M (2008) Application of internal reflux in the raffinate phase for membrane extraction in cross-flow rectangular modules. J Chin Inst Chem Eng 39:571-578. https://doi.org/10.1016/j.jcice.2008.05.010

Yeh HM, Chang YH (2005) Mass transfer for dialysis through parallelflow double-pass rectangular membrane modules. J Membr Sci 260:1-9. https://doi.org/10.1016/j.memsci.2005.03.003

Yeh H-M, Hsu Y-S (1999) Analysis of membrane extraction through rectangular mass exchangers. Chem Eng Sci 54:897-908. https:// doi.org/10.1016/S0009-2509(98)00272-3
Zhou H-X, Pang X (2018) Electrostatic interactions in protein structure, folding, binding, and condensation. Chem Rev 118:1691-1741. https://doi.org/10.1021/acs.chemrev.7b00305

Publisher's Note Springer Nature remains neutral with regard to jurisdictional claims in published maps and institutional affiliations. 